\title{
MINIMAL POSITIVE REALIZATIONS OF LINEAR CONTINUOUS-TIME FRACTIONAL DESCRIPTOR SYSTEMS: TWO CASES OF AN INPUT-OUTPUT DIGRAPH STRUCTURE
}

\author{
KONRAD ANDRZEJ MARKOWSKI ${ }^{a}$ \\ ${ }^{a}$ Institute of Control and Industrial Electronics, Faculty of Electrical Engineering \\ Warsaw University of Technology, Koszykowa 75, 00-662 Warsaw, Poland \\ e-mail: Konrad.Markowski@ee.pw.edu.pl
}

\begin{abstract}
In the last two decades, fractional calculus has become a subject of great interest in various areas of physics, biology, economics and other sciences. The idea of such a generalization was mentioned by Leibniz and L'Hospital. Fractional calculus has been found to be a very useful tool for modeling linear systems. In this paper, a method for computation of a set of a minimal positive realization of a given transfer function of linear fractional continuous-time descriptor systems has been presented. The proposed method is based on digraph theory. Also, two cases of a possible input-output digraph structure are investigated and discussed. It should be noted that a digraph mask is introduced and used for the first time to solve a minimal positive realization problem. For the presented method, an algorithm was also constructed. The proposed solution allows minimal digraph construction for any one-dimensional fractional positive system. The proposed method is discussed and illustrated in detail with some numerical examples.
\end{abstract}

Keywords: fractional system, positive systems, descriptor systems, realization, digraph structure, digraph mask, algorithm.

\section{Nomenclature}

A : matrices denoted in boldface,

$\mathbf{A}^{-1}$ : inverse matrix

$\operatorname{det}(\mathbf{A})$ : determinant of the matrix $\mathbf{A}$,

$\mathbf{A} \geq 0$ : non-negative matrix,

$\mathbb{A}:$ sets denoted by the double line,

$i$ : lower/upper indices and polynomial coefficients,

${ }_{a}^{C} \mathfrak{D}_{t}^{\alpha}$ : Caputo fractional derivative,

$\mathcal{D}$ : one-dimensional digraph,

$v_{i}$ : vertex of a graph,

$\left(v_{i}, v_{j}\right):$ arc of a digraph,

$w\left(v_{i}, v_{j}\right):$ weight of an arc,

$\mathfrak{M}(\mathcal{D})$ : digraph mask,

$\bigcup$ : operation composition relative to vertices,

$\circ$ : operation superposition,

$\mathbb{R}^{n \times m}:$ set $n \times m$ of real matrices,

$\mathbb{R}_{+}$: set of non-negative numbers,

$\mathbb{Z}_{+}$: set of non-negative integers,

$\mathbf{I}_{n}: n \times n$ identity matrix,

$\mathcal{L}\{f(t)\}: \mathcal{L}$-transform of the function $f(t)$,

$T(s)$ : transfer function of one variable $s$,

$p(s)$ : polynomial of the variable $s$.

\section{Introduction}

Descriptor non-positive and positive continuous-time systems have been considered in many papers and books (Luenberger, 1979; Lewis, 1984; 1986; Dai, 1989; Farina and Rinaldi, 2000; Kaczorek, 2001; Dodig and Stoi, 2009; Guang-Ren, 2010; Kaczorek and Sajewski, 2014). Matrix theory for the descriptor system has been presented by Luenberger (1979), Kublanovskaya (1983) as well as Horn and Johnson (1991). There are many problems associated with the analysis and synthesis of descriptor systems. One of the very important ones is the positive realization and minimal positive realization problem. In many research studies, we can find a constant matrix form, which satisfies the system described by the transfer function (Farina and Rinaldi, 2000; Benvenuti and Farina, 2004; Kaczorek, 2007; Guang-Ren, 2010; Sajewski, 2012; Kaczorek and Sajewski, 2014). In fact, there are many sets of matrices which fit into the system transfer function.

Integral and differential calculus of fractional order has become a subject of great interest in various areas of physics, biology, economics and other sciences. Fractional calculus is a generalization of traditional 
integer order integration and differentiation actions onto non-integer order. The idea of such a generalization was mentioned in 1695 by Leibniz and L'Hospital. The first definition of the fractional derivative was introduced by Liouville and Riemann at the end of the 19th century (Nishimoto, 1984). However, it was only in the late 1960 s, that this idea drew the attention of engineers. Fractional calculus was found to be a very useful tool for modelling the behaviour of many materials and systems. Mathematical fundamentals of the fractional calculus are given by Nishimoto (1984), Miller and Ross (1993), Podlubny (1999), Das (2011), Ortigueira (2011) as well as Kaczorek and Sajewski (2014). Some other applications of fractional-order systems can be found in the works of Ionescu et al. (2010), Magin et al. (2011), Kaczorek (2011), Petras et al. (2012), Vandoorn et al. (2013), Podlubny et al. (2014), Machado and Lopes (2015), Machado et al. (2015), Muresan et al. (2016a), Sikora (2016), or Muresan et al. (2016b).

The main purpose of this paper is to present a method based on one-dimensional digraph theory for computation of the set of a minimal positive realization. The problem is related to a one-dimensional continuous-time fractional positive descriptor system. A digraph structure corresponding to minimal realizations of the descriptor system is presented and discussed. In this paper, for the first time, a digraph mask is introduced and used. It is very important in the analysis of descriptor systems, with the use of digraph theory. By using the digraph mask, we can realize singularity in the system considered. It should be noted that there are many different possible structures of masks. Thus, we get a wider set of possible realizations. As a result of this discussion, sufficient conditions of digraph structures are established. Finally, we propose a procedure for determining the set of positive realizations in the class $\mathcal{K}_{1}$ of the digraph structure. The digraph classes of two-dimensional systems were presented by Hryniów and Markowski (2016a). Based on them, Markowski (2018) defined and presented in detail classes for one-dimensional systems. In the publications by Hryniów and Markowski (2016b), Markowski (2016; 2017a) or Markowski and Hryniów (2017a; 2017b), minimal realization of the continuous-time linear fractional system as a set of matrices $(\mathbf{A}, \mathbf{B}, \mathbf{C}, \mathbf{D})$ was determined. This work is the next step in the research on the determination of the set of the realization in the form $(\mathbf{E}, \mathbf{A}, \mathbf{B}, \mathbf{C})$ of positive continuous-time non-fractional and fractional descriptor systems by using digraph theory, which was started by Markowski (2017b; 2017c).

This work is organised as follows. Section 2 presents models and representations of fractional continuous-time systems, basic properties of the descriptor continuous-time linear system defined as the state-space representation, as well as some notation and basic definitions of digraph theory which will be used in further. In Section 3, we construct a method for determination of a minimal positive realization of the descriptor continuous-time system which consists of four parts: determination matrix $\mathbf{A}$ (Section 3.1) and matrix $\mathbf{E}$ (Section 3.2), choosing sets of the proper pairs (E, A) (Section 3.3) and determination matrices $\mathbf{B}$ and C (Section 3.4). Sufficient conditions of the digraph structure are also given. Finally, we demonstrate some numerical examples (Section 4), present concluding remarks, open problems and the related bibliography.

\section{Background}

2.1. Model and representation. The equation for a continuous-time dynamic system of a fractional order can be written as follows (Podlubny, 1999):

$$
\begin{aligned}
& H\left(\mathfrak{D}^{\alpha_{0} \alpha_{1} \ldots \alpha_{r}}\right)\left(y_{1}, y_{2}, \ldots, y_{l}\right) \\
&=G\left(\mathfrak{D}^{\beta_{0} \beta_{1} \ldots \beta_{q}}\right)\left(u_{1}, u_{2}, \ldots, u_{k}\right)
\end{aligned}
$$

where $y_{i}, u_{i}$ represent functions of time and $H(\cdot)$, $G(\cdot)$ are combinations of the fractional-order derivative operators. For the linear time-invariant single-variable case, we obtain the following equation:

$$
\underbrace{\left(\sum_{k=0}^{r} a_{k} \mathfrak{D}^{\alpha_{k}}\right)}_{H\left(\mathfrak{D}^{\left.\alpha_{0} \alpha_{1} \ldots \alpha_{r}\right)}\right.}(y(t))=\underbrace{\left(\sum_{k=0}^{q} b_{k} \mathfrak{D}^{\beta_{k}}\right)}_{G\left(\mathfrak{D}^{\left.\beta_{0} \beta_{1} \ldots \beta_{q}\right)}\right.}(u(t)),
$$

where $a_{k} \in \mathbb{R}$ and $b_{k} \in \mathbb{R}$.

Applying the Laplace transform to (1) with zero initial conditions, the input-output representation can be obtained. The fractional-order system as the transfer function has the following form:

$$
G(s)=\frac{b_{q} s^{\beta_{q}}+b_{q-1} s^{\beta_{q-1}}+\cdots+b_{0} s^{\beta_{0}}}{a_{r} s^{\alpha_{r}}+a_{r-1} s^{\alpha_{r-1}}+\cdots+a_{0} s^{\alpha_{0}}} .
$$

In the case of a commensurate-order system, the continuous-time transfer function (2) is given in the following form: $G(s)=\sum_{k=0}^{q} b_{k}\left(s^{\alpha}\right)^{k} / \sum_{k=0}^{r} a_{k}\left(s^{\alpha}\right)^{k}$, and can be considered a pseudo-rational function $H(\lambda)$ of the variable $\lambda=s^{\alpha}$ in the form

$$
H(\lambda)=\frac{\sum_{k=0}^{q} b_{k} \lambda^{k}}{\sum_{k=0}^{r} a_{k} \lambda^{k}}=\frac{b_{0}+b_{1} \lambda+\cdots+b_{q} \lambda^{q}}{a_{0}+a_{1} \lambda+\cdots+a_{r} \lambda^{r}} .
$$

2.2. Descriptor fractional systems. In this paper the following Caputo definition (Caputo, 1967) of the 
fractional derivative will be used:

$$
{ }_{a}^{C} \mathfrak{D}_{t}^{\alpha}=\frac{\mathrm{d}^{\alpha}}{\mathrm{d} t^{\alpha}}=\frac{1}{\Gamma(n-\alpha)} \int_{a}^{t} \frac{f^{(n)}(\tau)}{(t-\tau)^{\alpha+1-n}} \mathrm{~d} \tau,
$$

where $\alpha \in \mathbb{R}$ is the order of a fractional derivative, $f^{(n)}(\tau)=\mathrm{d}^{n} f(\tau) / \mathrm{d} \tau^{n}$ and $\Gamma(x)=\int_{0}^{\infty} e^{-t} t^{x-1} \mathrm{~d} t$ is the gamma function.

Consider the fractional descriptor continuous-time linear system

$$
\begin{aligned}
\mathbf{E}_{0} \mathfrak{D}_{t}^{\alpha} x(t) & =\mathbf{A} x(t)+\mathbf{B} u(t), \quad 0<\alpha<1, \\
y(t) & =\mathbf{C} x(t),
\end{aligned}
$$

where $x(t) \in \mathbb{R}^{n}, u(t) \in \mathbb{R}^{m}$ and $y(t) \in \mathbb{R}^{p}$ are the state, input and output vectors, respectively, and

$$
\mathbf{E} \in \mathbb{R}^{n \times n}, \quad \mathbf{A} \in \mathbb{R}^{n \times n}, \quad \mathbf{B} \in \mathbb{R}^{n \times m}, \quad \mathbf{C} \in \mathbb{R}^{p \times n} .
$$

It is assumed that $\operatorname{det} \mathbf{E}=0$ and the pencil of the system (5) is regular, that is,

$$
\operatorname{det}\left[\mathbf{E} s^{\alpha}-\mathbf{A}\right] \neq 0
$$

for some $z \in \mathbb{C}$ (where $\mathbb{C}$ is the field of complex numbers).

Definition 1. The system (5) is called a descriptor system if and only if $\operatorname{det} \mathbf{E}=0(\operatorname{rank} \mathbf{E}=r<n)$.

Definition 2. The fractional descriptor linear system (5) is called internally positive if and only if $x(t) \in \mathbb{R}_{+}^{n}$ and $y(t) \in \mathbb{R}_{+}^{p}$ for $t \geq 0$ for any admissible initial conditions $x_{0} \in \mathbb{R}_{+}^{n}$ and all inputs $u(t) \in \mathbb{R}_{+}^{m}$ for $t \geq 0$.

2.3. Digraphs. A directed graph (or just a digraph) $\mathcal{D}$ consists of a non-empty finite set $\mathbb{V}(\mathcal{D})$ of elements called vertices and a finite set $\mathbb{A}(\mathcal{D})$ of ordered pairs of distinct vertices called arcs (Bang-Jensen and Gutin, 2009). We call $\mathbb{V}(\mathcal{D})$ the vertex set and $\mathbb{A}(\mathcal{D})$ the arc set of digraph $\mathcal{D}$. We will often write $\mathcal{D}=(\mathbb{V}, \mathbb{A})$, which means that $\mathbb{V}$ and $\mathbb{A}$ are the vertex set and the arc set of $\mathcal{D}$, respectively. The order of $\mathcal{D}$ is the number of vertices in $\mathcal{D}$. The size of $\mathcal{D}$ is the number of $\operatorname{arcs}$ in $\mathcal{D}$. For an $\operatorname{arc}\left(v_{1}, v_{2}\right)$, the first vertex $v_{1}$ is its tail and the second vertex $v_{2}$ is its head.

There are two well-known methods of representation of a digraph: a list and an incidence matrix. In this paper we are using the incidence matrix to represent all digraphs. The manner of constructing digraphs with this method is presented, for example, by Godsil and Royle (2001) or Bang-Jensen and Gutin (2009).
Assume that the following matrices are given:

$(\mathbf{A}, \mathbf{B})$

$$
=\left(\begin{array}{cccc}
v_{i} \backslash^{v_{j}} & v_{1} & v_{2} & v_{3} \\
v_{1} \\
v_{2} \\
v_{3}
\end{array}\left[\begin{array}{ccc}
0 & 0 & 1 \\
1 & 0 & 1 \\
0 & 1 & 1
\end{array}\right], \quad \begin{array}{c}
v_{i} \backslash^{s_{m}} \\
v_{1} \\
v_{2} \\
v_{3}
\end{array}\left[\begin{array}{l}
s_{1} \\
1 \\
1 \\
0
\end{array}\right]\right) .
$$

There exists an arc from vertex $v_{j}$ to vertex $v_{i}$ (or from vertex $s_{m}$ to vertex $v_{i}$ ) if and only if the $(i, j)$-th (or $(i, m)$-th) entry of the matrix is non-zero. A one-dimensional digraph corresponding to the matrices (6) is presented in Fig. 1

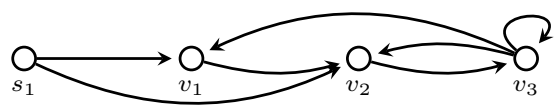

Fig. 1. One-dimensional digraph of (6).

We present below some basic notions from graph theory which are used in our further discussion. A walk in a digraph $\mathcal{D}$ is a finite sequence of arcs in which every two vertices $v_{i}$ and $v_{j}$ are adjacent or identical. A walk in which all of the arcs are distinct is called a path. The path that goes through all vertices is called a finite path. If the initial and terminal vertices of the path are the same, then the path is called a cycle. One of the very important operations on digraphs is composition relative to vertices.

Definition 3. (Composition relative to vertices) Let $\mathcal{G}_{1}, \mathcal{G}_{2}, \ldots, \mathcal{G}_{n}$ be digraphs with vertex sets $\mathbb{V}\left(\mathcal{G}_{n}\right)=$ $\left\{v_{i(n)}: i \in \mathbb{N}\right\}$. The composition relative to vertices $\mathcal{D}\left[\mathcal{G}_{1}, \mathcal{G}_{2}, \ldots, \mathcal{G}_{n}\right]$ is the digraph $\mathcal{L}$ with the vertex set $\mathbb{V}\left(\mathcal{G}_{1}\right) \cup \mathbb{V}\left(\mathcal{G}_{2}\right) \cup \cdots \cup \mathbb{V}\left(\mathcal{G}_{n}\right)=\left\{v_{j}: j=\right.$ $1, \ldots, \max \{i(n)\}, i \in \mathbb{N}\}$ and arc set $\mathcal{A}^{\prime}=A\left(\mathcal{G}_{1}\right) \cup$ $A\left(\mathcal{G}_{2}\right) \cup \cdots \cup A\left(\mathcal{G}_{n}\right)$, where $A^{\prime}$ denotes the operation of deleting multiple arcs.

More information on the use of digraph theory in analysis of positive and non-positive system is given by Fornasini and Valcher (1997; 2005), Godsil and Royle (2001) or Bang-Jensen and Gutin (2009).

\subsection{Problem formulation.}

Theorem 1. (Podlubny, 1999) The Laplace transform of the derivative-integral (4) has the form

$$
\mathcal{L}\left[\begin{array}{l}
C \\
0
\end{array} \mathfrak{D}_{t}^{\alpha}\right]=s^{\alpha} F(s)-\sum_{k=1}^{n} s^{\alpha-k} f^{(k-1)}\left(0^{+}\right) .
$$

After using the Laplace transform in (5), Theorem 1 
and taking into account that

$$
\begin{aligned}
X(s) & =\mathcal{L}[x(t)]=\int_{0}^{\infty} x(t) e^{-s t} \mathrm{~d} t, \\
\mathcal{L}\left[\mathfrak{D}^{\alpha} x(t)\right] & =s^{\alpha} X(s)-s^{\alpha-1} x_{0},
\end{aligned}
$$

we obtain

$$
\begin{aligned}
& X(s)=\left[\mathbf{E} s^{\alpha}-\mathbf{A}\right]^{-1}\left[s^{\alpha-1} x_{0}+\mathbf{B} U(s)\right], \\
& Y(s)=\mathbf{C} X(s), \quad U(s)=\mathcal{L}[u(t)] .
\end{aligned}
$$

From (7) we can determine the transfer matrix of the system in the following form:

$$
\mathbf{T}(s)=\mathbf{C}\left[\mathbf{E} s^{\alpha}-\mathbf{A}\right]^{-1} \mathbf{B} \in \mathbb{R}^{p \times m}(s) .
$$

Definition 4. (Berman and Plemmons, 1979) A matrix $\mathbf{M}=\left[m_{i, j}\right] \in \mathbb{R}^{n \times n}$ is called a Metzler matrix if its off-diagonal entries are non-negative, $m_{i j} \geq 0$.

As has been shown by Mitkowski (2008), every non-negative matrix is a Metzler matrix. For every Metzler matrix there exists a real number $\eta \geq \min _{i} m_{i j}$ such that $\eta \mathbf{I}+\mathbf{M}=\mathbf{A}$ is a non-negative matrix.

The descriptor continuous-time linear system (5) is positive if and only if

$$
\mathbf{E} \in \mathbb{R}_{+}^{n \times n}, \quad \mathbf{A} \in \mathbb{M}_{n}, \quad \mathbf{B} \in \mathbb{R}_{+}^{n \times m}, \quad \mathbf{C} \in \mathbb{R}_{+}^{p \times n} .
$$

The matrices (9) are called a realization of a given transfer matrix $\mathbf{T}(s) \in \mathbb{R}^{p \times m}(s)$ if they satisfy the equality (8). The realization $(\mathbf{E}, \mathbf{A}, \mathbf{B}, \mathbf{C})$ is called a minimal realization if the dimension of the state matrix $\mathbf{A}$ is minimal among all possible realizations of $\mathbf{T}(s)$.

The task is as follows: For the given transfer matrix (8), determine a minimal positive realization of the fractional descriptor continuous-time system (5) using one-dimensional $\mathcal{D}$ digraph theory. The dimension of the system must be minimal among all possible ones.

\section{Problem solution}

The solution to the minimal positive realization problem will be presented for a one-dimensional single-input single-output (SISO) system for $m=p=1$. The proposed method will be based on one-dimensional digraph theory, and it will determine solutions in class $\mathcal{K}_{1}$. The classes of the digraph structure are considered in detail by Markowski (2018).

Consider the irreducible transfer function

$$
T(s)=\frac{n(s)}{d(s)}
$$

with

$$
\begin{aligned}
n(s)= & b_{q}\left(s^{\alpha}\right)^{q}+b_{q-1}\left(s^{\alpha}\right)^{(q-1)}+b_{q-2}\left(s^{\alpha}\right)^{(q-2)} \\
& +\cdots+b_{1} s^{\alpha}+b_{0}, \\
d(s)= & a_{r}\left(s^{\alpha}\right)^{r}+a_{r-1}\left(s^{\alpha}\right)^{(r-1)}+a_{r-2}\left(s^{\alpha}\right)^{(r-2)} \\
& +\cdots+a_{1} s^{\alpha}+a_{0},
\end{aligned}
$$

where $b_{i}, i=0,1, \ldots, q$ and $a_{j}, j=0,1, \ldots, r$ are given real coefficients, and $r<q$. We can rewrite the transfer function (10) in the form (3). In this case, the transferred function can be considered a pseudo-rational function of the variable $\lambda=s^{\alpha}$ in the form

$$
T(\lambda)=\frac{n(\lambda)}{d(\lambda)}
$$

with

$$
\begin{aligned}
n(\lambda)= & b_{q} \lambda^{q}+b_{q-1} \lambda^{q-1}+b_{q-2} \lambda^{q-2} \\
& +\cdots+b_{1} \lambda+b_{0}, \\
d(\lambda)= & a_{r} \lambda^{r}+a_{r-1} \lambda^{r-1} a_{r-2} \lambda^{r-2} \\
& +\cdots+a_{1} \lambda+a_{0} .
\end{aligned}
$$

The proposed method consists of four parts:

- In Section 3.1, we determine state matrices A with the use of digraph theory. The characteristic polynomial $d(\lambda)$ is considered in the temporary form $\widetilde{d}(\lambda)$ which is needed to determine a digraph structure. It should be noted that this temporary form does not change coefficients and powers of the characteristic polynomial $d(\lambda)$.

- In Section 3.2, we determine matrix $\mathbf{E}$ using digraph mask $\mathfrak{M}(\mathcal{D})$.

- Using the digraph structure determined in Section 3.1 and the digraph mask determined in Section 3.2. we can create (Section 3.3) all possible realizations as combinations of the characteristic polynomial realizations and a digraph mask. After this operation, we obtain the set of matrix pairs $(\mathbf{E}, \mathbf{A})$. It should be noted that after using the digraph mask we obtain the characteristic polynomial in the desired form $d(\lambda)$.

- Finally, in Section 3.4, we should determine matrices $\mathbf{B}$ and $\mathbf{C}$ by extending the digraphs obtained in Section 3.3 Here we will consider two possible cases (Sections 3.4.1 and 3.4.2) of the digraph structure.

3.1. Determination of state matrix $\mathbf{A}$. In the first step, we must find matrix A. After multiplying the denominator of a transfer matrix (11) by $\lambda^{-(q+1)}$, we 
obtain the following characteristic polynomial:

$$
\begin{aligned}
d(\lambda)= & a_{r} \lambda^{r-q-1}+a_{r-1} \lambda^{r-q-2} \\
& +a_{r-2} \lambda^{r-q-3}+\cdots+a_{1} \lambda^{-q}+a_{0} \lambda^{-q-1} .
\end{aligned}
$$

In order to determine the digraph structure corresponding to the characteristic polynomial (12), we must present them in the following temporary form, which is needed to draw the digraphs:

$$
\begin{aligned}
\tilde{d}(\lambda)= & 1+d(\lambda) \\
= & 1+a_{r} \lambda^{r-q-1}+a_{r-1} \lambda^{r-q-2} \\
& +a_{r-2} \lambda^{r-q-3}+\cdots+a_{1} \lambda^{-q}+a_{0} \lambda^{-q-1} .
\end{aligned}
$$

Remark 1. It should be noted that after this operation we can consider a characteristic polynomial of the descriptor system in the same way as a characteristic polynomial of the standard system thus $\mathbf{E} \Rightarrow \mathbf{I}$ (Layer 1 in Fig. 2). In the next steps (Section 3.2), we introduce a digraph mask $\mathfrak{M}(\mathcal{D})$ (Layer 2 in Fig. 2), which guarantees the singularity of the characteristic polynomial thus $\mathbf{I} \Rightarrow \mathbf{E}$ (Layer 3 in Fig. 2).

Then we turn the decomposition polynomial (13) into a set of binomials:

$$
\begin{aligned}
& \tilde{d}(\lambda) \\
& =\underbrace{\left(1-a_{r} \lambda^{r-q-1}\right)}_{B_{r}} \cup \underbrace{\left(1-a_{r-1} \lambda^{r-q-2}\right)}_{B_{r-1}} \\
& \cup \underbrace{\left(1-a_{r-2} \lambda^{r-q-3}\right)}_{B_{r-2}} \cup \ldots \cup \underbrace{\left(1-a_{1} \lambda^{-q}\right)}_{B_{1}} \\
& \cup \underbrace{\left(1-a_{0} \lambda^{-q-1}\right)}_{B_{0}} \\
& =\bigcup_{i=0}^{r}\left(1-a_{r} \lambda^{i-q-1}\right)=\bigcup_{i=0}^{r} B_{i},
\end{aligned}
$$

where $r$ is a number of binomials in the characteristic polynomial and $U$ is the operation called a composition relative to vertices in digraphs presented in Definition 3 .

For each binomial (14), using the following result, we create digraph representations.

Proposition 1. (Binomial digraph representation) $\mathrm{Di}$ graph $\mathcal{D}$ corresponding to binomial $1-a_{r} \lambda^{r-q-1}$, where $r=0,1, \ldots \ldots, q-1$, consists of one cycle, and it contains $|r-q-1|$-vertices.

Proof. Suppose that the digraph consists of the vertices $v_{1}, v_{2}, \ldots, v_{q}, v_{q+1}$. Assume that each of the arcs in the digraph we assigned weights in the form of $w\left(v_{i}, v_{j}\right) \lambda^{-1}$ for $i, j \in \mathbb{Z}_{+}=\{1,2, \ldots, q+1\}$, where $v_{i}$ is the tail and $v_{j}$ is the head, and $i \neq j$. Consider the boundary points in the set:

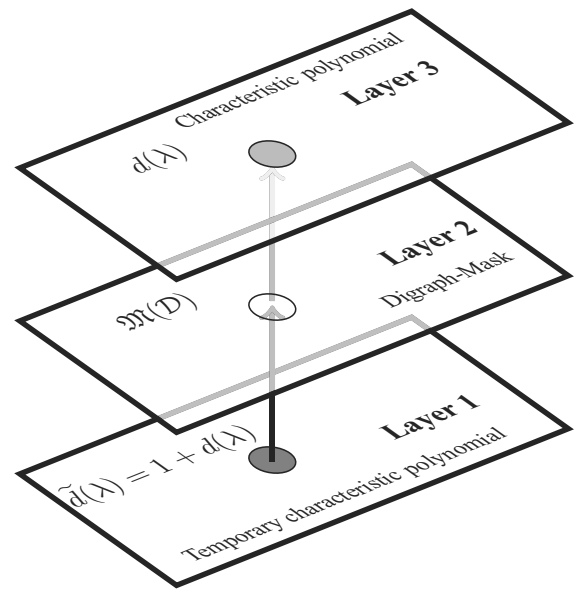

Fig. 2. Relation between $d(\lambda), \widetilde{d}(\lambda)$ and $\mathfrak{M}(\mathcal{D})$.

- $r=q-1 \Rightarrow$ power of the binomial $1-a_{r} \lambda^{r-q-1}$ is equal to $a_{q-1} \lambda^{-2}$. The cycle can be written as $w\left(v_{i}, v_{j}\right) \lambda^{-1} \cdot w\left(v_{j}, v_{i}\right) \lambda^{-1}$. Therefore, the maximum weight in a digraph is equal to $\left[w\left(v_{i}, v_{j}\right)\right.$. $\left.w\left(v_{j}, v_{i}\right)\right] \lambda^{-2}=a_{q-1} \lambda^{-2}$.

- $r=0 \Rightarrow$ power of the binomial $1-a_{r} \lambda^{r-q-1}$ is equal to $a_{0} \lambda^{-(q+1)}$. In this case the cycle can be written as $w\left(v_{1}, v_{2}\right) \lambda^{-1} \cdot w\left(v_{2},:\right) \lambda^{-1} \cdots \cdots w($ : , $\left.v_{i}\right) \lambda^{-1} w\left(v_{i}, v_{j}\right) \lambda^{-1} \cdot w\left(v_{j}, v_{1}\right) \lambda^{-1}$, where ' $:$ ' is the next vertex in the cycle. Because $i, j=q+1$ and $i \neq j$, the maximum weight in a digraph is equal to $\left[w\left(v_{1}, v_{2}\right) \cdot w\left(v_{2},:\right) \cdot \ldots \cdot w\left(:, v_{i}\right) \cdot w\left(v_{i}, v_{j}\right)\right.$. $\left.w\left(v_{j}, v_{1}\right)\right] \lambda^{-(q+1)}=a_{0} \lambda^{-(q+1)}$.

Using Proposition 1, Theorem 1 presented by Hryniów and Markowski $(2014 ; 2015)$ and Definition 3 , we can create all digraph realizations of the characteristic polynomials in a temporary form (13). Each digraph corresponding to a characteristic polynomial must satisfy two conditions. The first condition $(\mathrm{C} 1)$ is related to the existence of the common part of the digraph (vertex in dark gray), the second condition (C2) is related to the non-existence of additional cycles in the digraph. To the vertices belonging to the common part of the digraph we assigned weight equal to 1 and to the other vertices-weight equal to 0 . Then we must determine arc weights which appear in the obtained digraphs corresponding to the temporary polynomial (13).

One of the possible digraph structures, for the power of the nominator equal to $q$ and the power of the denominator equal to $r=q-1$, is presented in Fig. 3(a).

Remark 2. Other realizations can be obtained from the digraph presented in Fig. 3(a) by the following actions:

- Renumbering vertices in the digraph. This operation is similar to circle, rotation and synonymous with 


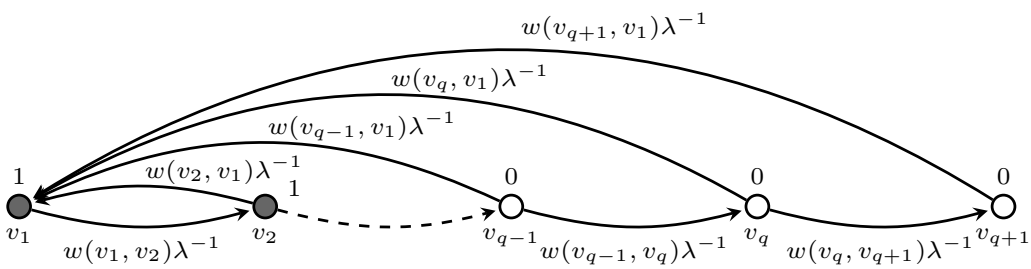

(a)

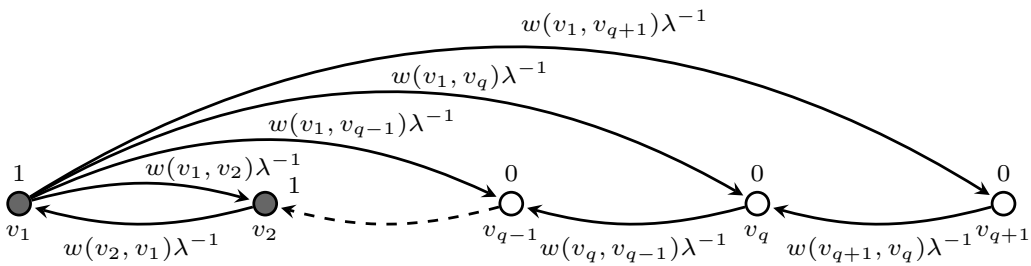

(b)

Fig. 3. Possible realization of the characteristic polynomial (13).

swapping columns / rows of a matrix used in matrix theory. The result of this operation is presented in Fig. 4.

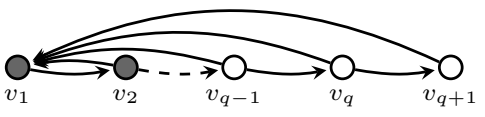

(a)

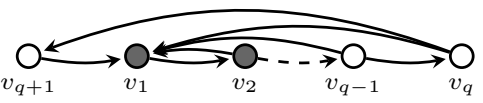

(b)

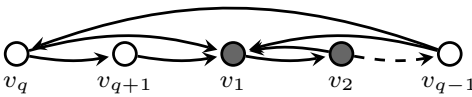

(c)

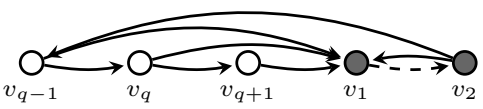

(d)

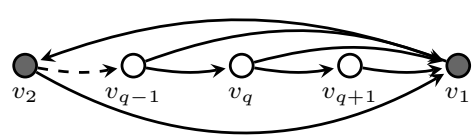

(e)

Fig. 4. Possible realization of the characteristic polynomial (13) obtained by renumbering vertices.

- Change of the direction of the arcs in the graph. This operation is similar to a transposition matrix used in matrix theory. The result of this operation is presented in Fig. 3(b).

Let us consider the following simple example.

Example 1. (Determination of a digraph structure corresponding to the characteristic polynomial) The characteristic polynomial $d(\lambda)= \pm \lambda^{-2} \pm \lambda^{-3}$ is rewritten in the temporary form $\widetilde{d}(\lambda)=1+d(\lambda)=1 \pm \lambda^{-2} \pm \lambda^{-3}$, consisting of two binomials: $B_{1}=1 \pm \lambda^{-2}$ and $B_{0}=1 \pm$ $\lambda^{-3}$. Possible realizations of the binomials are presented in Fig. 5 .

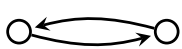

(a)

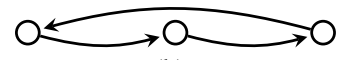

(b)
Fig. 5. Realization of the binomial: $B_{1}=1 \pm \lambda^{-2}$ (a), $B_{0}=$ $1 \pm \lambda^{-3}$ (b)

Then, we can determine all possible realizations (Fig. 6) of the polynomial $\widetilde{d}(\lambda)$ which satisfy conditions (C1) and (C2). Additionally, to the vertices belonging to the common part of the digraph we assigned weight equal to 1 (marked in dark gray) and 0 for other vertices.

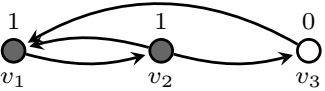

(a)

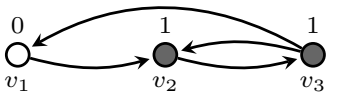

(b)

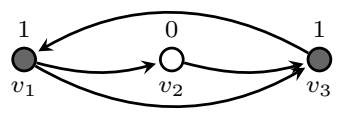

(c)

Fig. 6. All possible realizations of the characteristic polynomial $\widetilde{d}(\lambda)$.

In the last step, we determine weights of the arcs by solving a set of equations. For example, from the digraph presented in Fig. 6(b), we obtain

$$
\left\{\begin{array}{l}
w\left(v_{1}, v_{2}\right) \cdot w\left(v_{2}, v_{3}\right) \cdot w\left(v_{3}, v_{1}\right)=\mp 1 \\
w\left(v_{2}, v_{3}\right) \cdot w\left(v_{3}, v_{2}\right)=\mp 1,
\end{array}\right.
$$


with the constraints $w\left(v_{1}, v_{2}\right)>0, w\left(v_{2}, v_{3}\right)>0$, $w\left(v_{3}, v_{2}\right)>0$ and $w\left(v_{3}, v_{1}\right)>0$ which must be satisfied if the realization is positive.

3.2. Determination of matrix E. In the second step, we must find matrix $\mathbf{E}$. The weight of the vertices in a digraph is associated with the digraph mask. If the characteristic polynomial is in the form of (13), then we can determine the digraph mask with the following proposition.

Proposition 2. (Digraph mask) A digraph mask $\mathfrak{M}(\mathcal{D})$ corresponding to the characteristic polynomial (13) consists of $q+1$ vertices, and at least one vertex in the digraph must have zero weight.

Proof. If a digraph mask $\mathfrak{M}(\mathcal{D})$ consists of all vertices with non-zero weights, this means that matrix $\mathbf{E}$ is diagonal with all non-zero entries, so that $\mathbf{E}=\mathbf{I}$. In this case, the condition $\operatorname{det} \mathbf{E}=0$ is not satisfied and the system is not a descriptor. There appears at least one zero entry guaranteeing that the condition $\operatorname{det} \mathbf{E}=0$ is satisfied and the system (5) is a descriptor.

All possible digraph masks of the characteristic polynomial (13) are presented in Fig. 7) It should be noted that vertices with weights equal to 1 are marked in dark gray and the other vertices have zero weights.

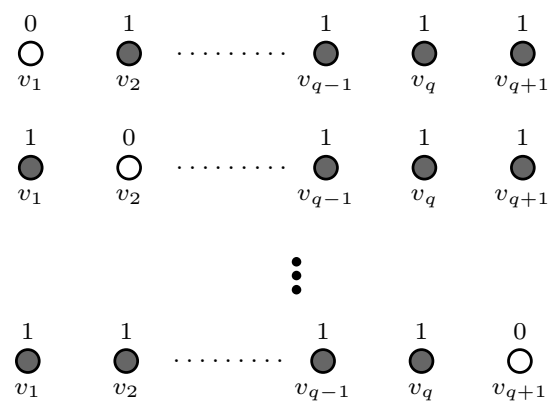

Fig. 7. All possible digraph masks of the characteristic polynomial (13).

Example 2. (Determination of a digraph mask-Example 1 continued) After using Proposition 2 , we obtain the set of digraph masks (presented in Fig. 8) consisting of two vertices with weight equal to 1 .

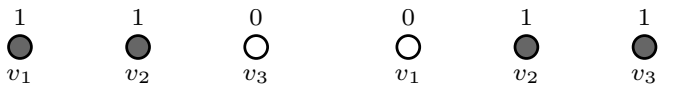

(a)

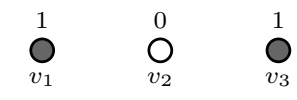

(c)

Fig. 8. All possible digraph masks of the characteristic polynomial $\widetilde{d}(\lambda)=1 \pm \lambda^{-2} \pm \lambda^{-3}$.
3.3. Choosing the proper pairs $(\mathbf{E}, \mathbf{A})$. With the use of Definition 3 and a digraph mask determined by Proposition 2, we can create all possible digraph realizations as combinations of the binomial representations and a digraph mask:

$$
\begin{aligned}
{ }_{k}^{d(\lambda)} \mid \mathcal{D} & =\mathfrak{M}_{j}(G) \circ\left(\mathcal{G}_{B_{r}} \cup \mathcal{G}_{B_{r-1}} \ldots \mathcal{G}_{B_{1}} \cup \mathcal{G}_{B_{0}}\right) \\
& =\mathfrak{M}_{j}(G) \circ\left(\bigcup_{i=0}^{r} \mathcal{G}_{B_{i}}\right)
\end{aligned}
$$

where ${ }_{k}^{d(\lambda)} \mid \mathcal{D}$ means that we consider the $k$-th realization of the one-dimensional digraph $\mathcal{D}$ corresponding to the characteristic polynomial $d(\lambda), \mathfrak{M}_{j}(G)$ is the $j$-th possible digraph masks, $\circ$ is the operation of the superposition of the digraph mask and one of the possible digraph realizations. It should be noted that the superposition $\circ$ on digraph vertices corresponds to the logical operation OR. We choose a digraph structure in which all vertices have weights equal to 1 . Other solutions are rejected. Let us consider the following simple example.

Example 3. (Determined pairs (E, A)-Example 2 continued) Using all possible realizations of the temporary polynomial $\widetilde{d}(\lambda)=1 \pm \lambda^{-2} \pm \lambda^{-3}$ presented in Fig. 6, a digraph mask presented in Fig. 8 and (15), we can create all possible digraph realizations which satisfy a characteristic polynomial $d(\lambda)= \pm \lambda^{-2} \pm \lambda^{-3}$. In Fig. 9 we present a realization for a digraph structure from Fig. 6(a) It should be noted that the digraphs presented in Figs. 9(f) and 9(i) have all vertices with weights equal to 1 and these digraph structures are correct. In the same way, we proceed with other structures from Figs. 6(b) and (c). Finally, we obtain six possible realizations.

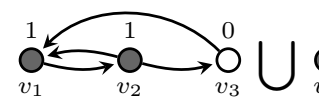

(a)

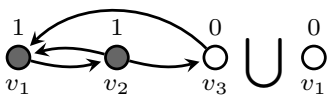

(d)

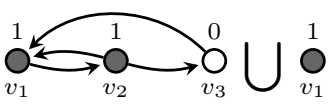

(g)

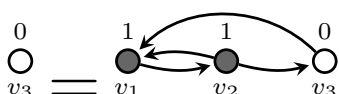

(c) (b)

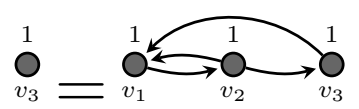

(e)

(f)

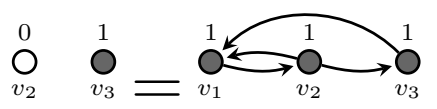

(h)

(i)
Fig. 9. All possible realizations of the characteristic polynomial $d(\lambda)= \pm \lambda^{-2} \pm \lambda^{-3}$.

Now we can write a matrix pair $\left(\mathbf{E}_{1}, \mathbf{A}\right)$ and $\left(\mathbf{E}_{2}, \mathbf{A}\right)$ corresponding to the digraph from Figs. 9(f) and (i), 


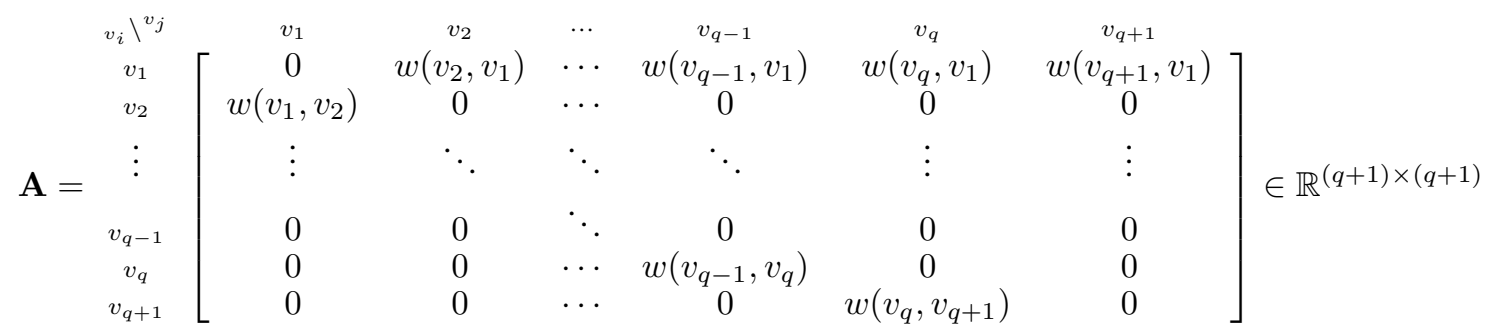

$$
\begin{aligned}
& \widetilde{\mathbf{A}}=\begin{array}{c}
v_{i}{ }^{v_{j}} \\
v_{1} \\
v_{2} \\
\vdots \\
v_{q-1} \\
v_{q} \\
v_{q+1}
\end{array}\left[\begin{array}{cccccc}
v_{2} & \cdots & v_{q-1} & v_{q} & v_{q+1} \\
w\left(v_{1}, v_{2}\right) & 0 & \cdots & 0 & 0 & 0 \\
\vdots & \ddots & \ddots & \ddots & \vdots & \vdots \\
w\left(v_{1}, v_{q-1}\right) & 0 & \ddots & 0 & w\left(v_{q}, v_{q-1}\right) & 0 \\
w\left(v_{1}, v_{q}\right) & 0 & \cdots & 0 & 0 & w\left(v_{q+1}, v_{q}\right) \\
w\left(v_{1}, v_{q+1}\right) & 0 & \cdots & 0 & 0 & 0
\end{array}\right] \in \mathbb{R}^{(q+1) \times(q+1)}
\end{aligned}
$$

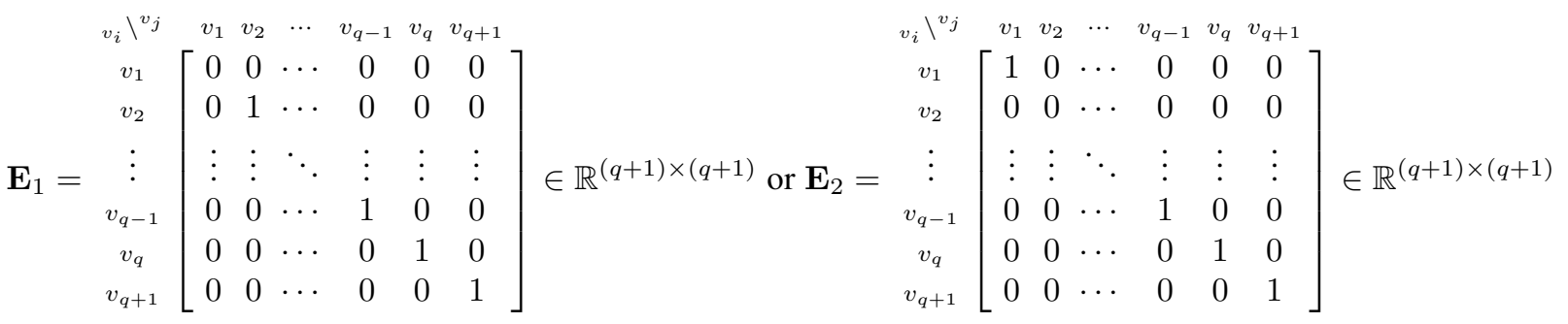

respectively, in the following form:

$$
\begin{aligned}
& \left(\mathbf{E}_{1}, \mathbf{A}\right)=\left(\left[\begin{array}{lll}
0 & 0 & 0 \\
0 & 1 & 0 \\
0 & 0 & 1
\end{array}\right],\left[\begin{array}{lll}
0 & 1 & 1 \\
1 & 0 & 0 \\
0 & 1 & 0
\end{array}\right]\right), \\
& \left(\mathbf{E}_{2}, \mathbf{A}\right)=\left(\left[\begin{array}{lll}
1 & 0 & 0 \\
0 & 0 & 0 \\
0 & 0 & 1
\end{array}\right],\left[\begin{array}{lll}
0 & 1 & 1 \\
1 & 0 & 0 \\
0 & 1 & 0
\end{array}\right]\right) .
\end{aligned}
$$

In the general form, one of the possible digraph structures corresponds to the temporary polynomial (13) presented in Fig. 3(a) With the use of the digraph mask presented in Fig. 7 we can create all possible realizations which satisfy the characteristic polynomial (12). It should be noted that we always have minimum two possible realizations because two vertices belong to digraph common parts. Now we can write the set of matrices $\left(\mathbf{E}_{1}, \mathbf{A}\right)$ and $\left(\mathbf{E}_{2}, \mathbf{A}\right)$ in the form (16) and (18).

Remark 3. If we change the direction of the arcs in the digraph presented in Fig. 3(a), we obtain the digraph presented in Fig. 3(b) This operation is similar to the transposition of the matrix $\mathbf{A}$. Then, we can write the set of the matrices $\left(\mathbf{E}_{1}, \widetilde{\mathbf{A}}\right)$ and $\left(\mathbf{E}_{2}, \widetilde{\mathbf{A}}\right)$ in the form of 17 and (18).

From the above discussion we can write the following proposition.

Proposition 3. (State matrix entries) The system (5) with matrices of the form (16) (or 17) is positive if and only if the arc weight in a digraph satisfy the condition $w\left(v_{i}, v_{j}\right) \geq 0$ for $i, j \in \mathbb{Z}_{+}=\{1,2, \ldots, q+1\}$ and $i \neq j$.

3.4. Determination of matrices $B$ and $C$. In the last step, we must find matrices $\mathbf{B}$ and $\mathbf{C}$. After multiplying the numerator of the transfer function (11) by $\lambda^{-q}$, we obtain the following polynomial:

$$
\begin{aligned}
n(\lambda)= & b_{q}+b_{q-1} \lambda^{-1}+b_{q-2} \lambda^{-2} \\
& +\cdots+b_{1} \lambda^{1-q}+b_{0} \lambda^{-q} .
\end{aligned}
$$

We can determine two possible digraph structures corresponding to the polynomial (19). Below we discuss this in detail. It should be noted that these cases are discussed in terms of a digraph structure resulting from the input and output connection. 


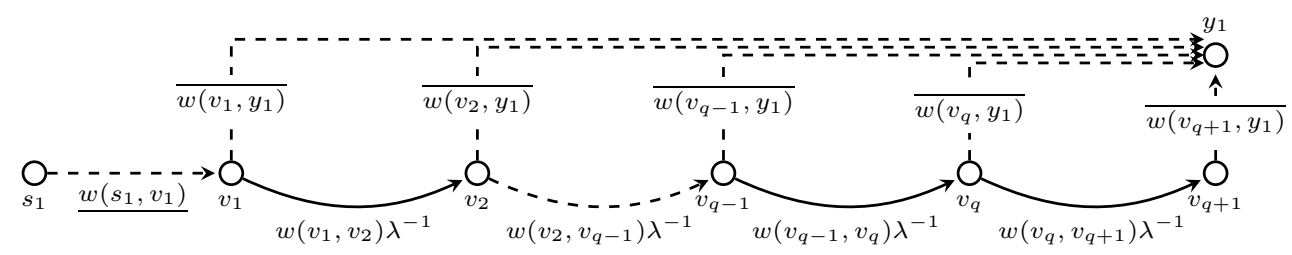

(a)

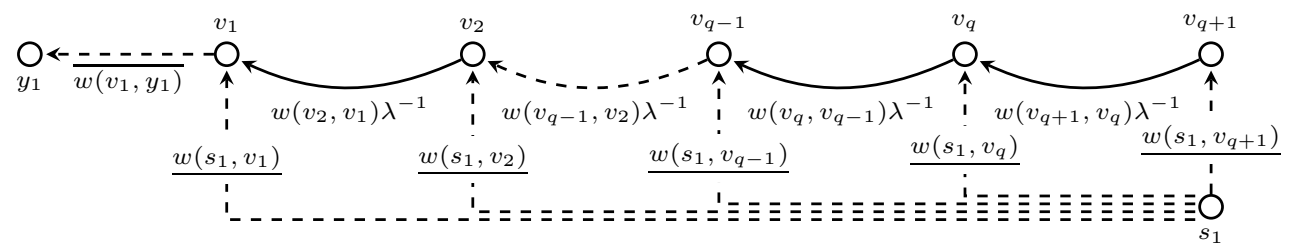

(b)

Fig. 10. Digraph corresponding to polynomial (19): Case 1 (a), Case 2 (b).

3.4.1. Case 1. For this purpose, we are expanding the digraph (see Fig. 3(a) created in the first part of the algorithm. We add the source vertex $s_{1}$ corresponding to matrix $\mathbf{B}$ and the output vertex $y_{1}$ corresponding to matrix C. We connect the source vertex $s_{1}$ with vertices $v_{1}$ or $v_{2}$ which belong to the common part of the digraph, and vertices $v_{1}, v_{2}, \ldots, v_{q}, v_{q+1}$ with the output vertex $y_{1}$. Then we determine all paths $p$ from the source vertex $s_{1}$ to the output vertex $y_{1}$. It should be noted that paths have length $2 \leq p \leq q+2$.

We obtain the digraph structure presented in Fig. 10(a) from which we can write the set of equations (20). After solving them we can write $\mathbf{B} \in \mathbb{R}^{(q+1) \times 1}$ and $\mathbf{C} \in \mathbb{R}^{1 \times(q+1)}$ in the form $22 \mathrm{a}$ ).

The positive realization $\left(\mathbf{E}_{1}, \mathbf{A}, \mathbf{B}, \mathbf{C}\right)$ of (11) is given by (16), 18) and (22a) if Proposition 3 applies and $w\left(s_{1}, v_{1}\right)>0$.

Remark 4. If we connect source $s_{1}$ with vertex $v_{2}$, then we must determine a new digraph structure, write the corresponding set of equations and solve them. Afterwards we can write matrices $\widetilde{\mathbf{B}}$ and $\widetilde{\mathbf{C}}$. In this case, the positive realization is given by $\left(\mathbf{E}_{2}, \mathbf{A}, \widetilde{\mathbf{B}}, \widetilde{\mathbf{C}}\right)$.

3.4.2. Case 2. For this purpose, we are expanding the digraph presented in Fig. 3(b), We add the source vertex $s_{1}$ corresponding to matrix $\mathbf{B}$ and the output vertex $y_{1}$ corresponding to matrix $\mathbf{C}$. We connect the source vertex $s_{1}$ with vertices $v_{1}, v_{2}, \ldots, v_{q}, v_{q+1}$, and vertices $v_{1}, v_{2}$ which belong to the common part of the digraph with the output vertex $y_{1}$. Then we determine all paths $p$ from the source vertex $s_{1}$ to the output vertex $y_{1}$. It should be noted that paths have length $2 \leq p \leq q+2$.

We obtain the digraph structure presented in Fig. 10(b) from which we can write the set of the equations (21) and after solving them we can write $\mathbf{B} \in$ $\mathbb{R}^{(q+1) \times 1}$, and $\mathbf{C} \in \mathbb{R}^{1 \times(q+1)}$ in the form of $\left.22 \mathrm{~b}\right)$.

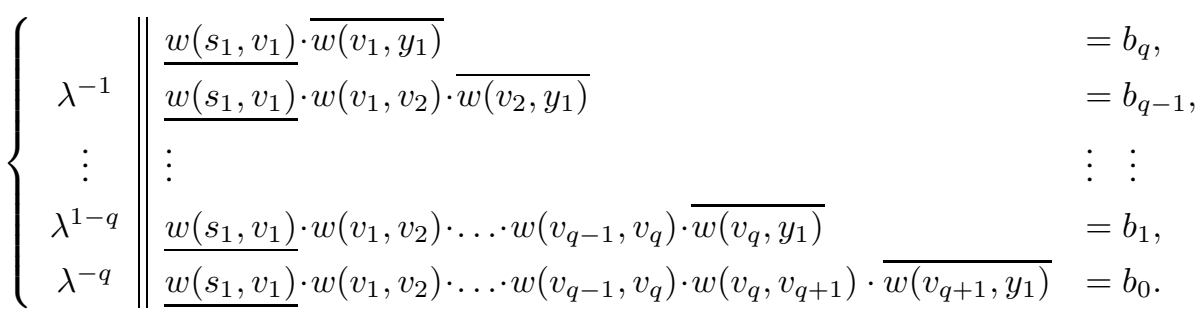

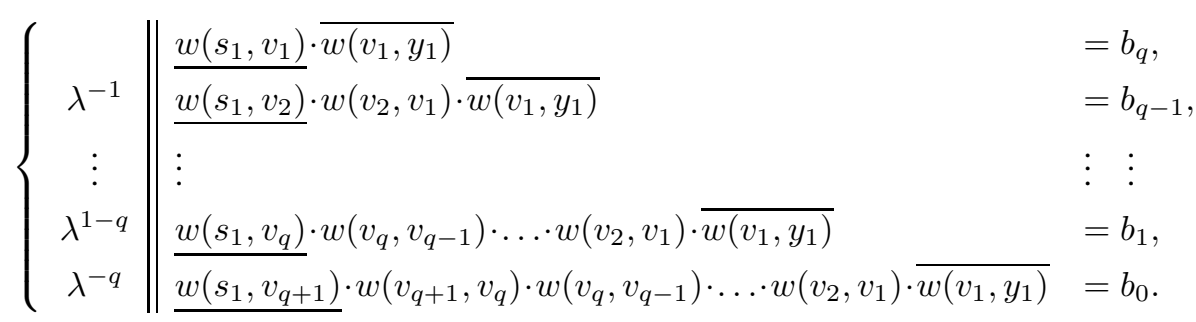




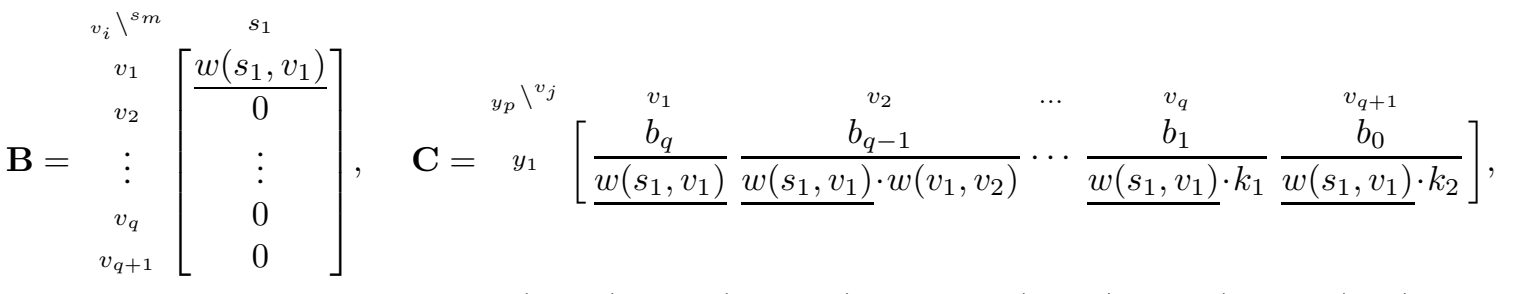

$$
\begin{aligned}
& k_{1}=w\left(v_{1}, v_{2}\right) \cdot \ldots \cdot w\left(v_{q-1}, v_{q}\right), \quad k_{2}=w\left(v_{1}, v_{2}\right) \cdot \ldots \cdot w\left(v_{q-1}, v_{q}\right) \cdot w\left(v_{q}, v_{q+1}\right)
\end{aligned}
$$

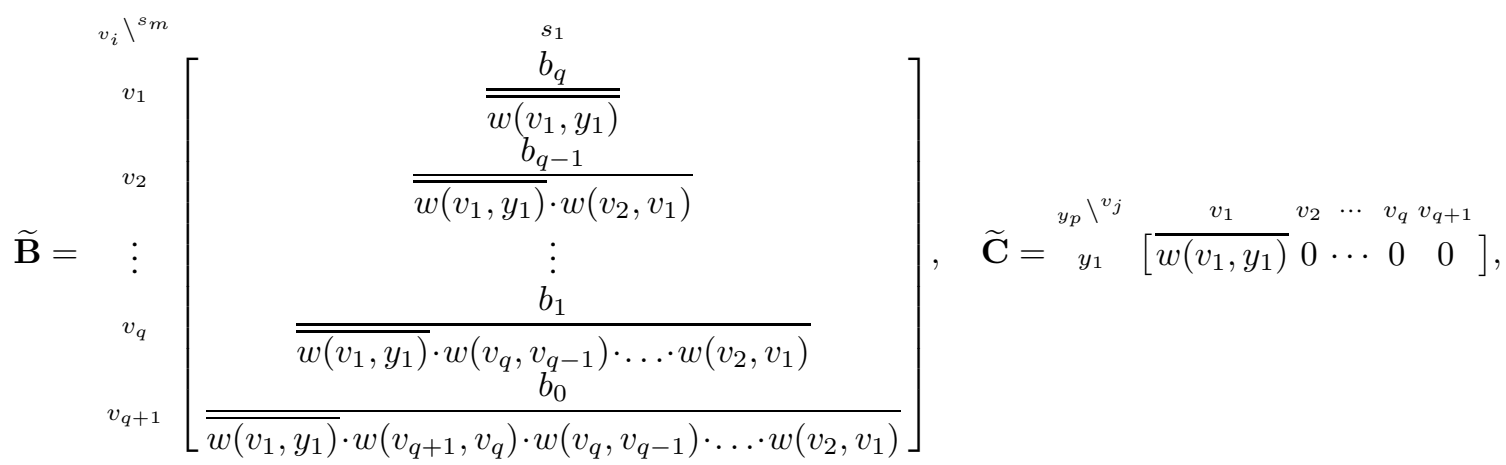

The positive realization $\left(\mathbf{E}_{1}, \widetilde{\mathbf{A}}, \widetilde{\mathbf{B}}, \widetilde{\mathbf{C}}\right)$ of 11 is given by (17), (18) and (22b) if Proposition 3 applies and $\overline{w\left(v_{1}, y_{1}\right)}>0$. As in Case 1, Remark 4 is correct as well.

From the above discussion (Cases 1 and 2), we can formulate the following result.

Proposition 4. (Input and output matrix entries) The system (5) with matrices of the form (22a) (or (22b) is positive if and only if the arc weights in the digraph satisfy the condition $w\left(s_{1}, v_{i}\right)>0$ and/or $\overline{w\left(v_{i}, y_{1}\right)}>0$ for $i \in \mathbb{Z}_{+}=\{1, \overline{2, \ldots, q+1}$.

Remark 5. It should be noted here that the digraphs presented in Cases 1 and 2 have simple structures and we have almost immediately to write a set of matrices $(\mathbf{E}, \mathbf{A}, \mathbf{B}, \mathbf{C})$. In other digraph structures this is not that immediate and additional conditions must be satisfied (see Section 4).

From the discussion led in Sections 3.1 3.4, we can write down the following sufficient conditions for digraph structures.

Proposition 5. There exists a digraph structure in class $\mathcal{K}_{1}$, which corresponds to a realization of a transfer function, if the following conditions are satisfied:

(C1) in the digraph corresponding to the characteristic polynomial there exists a non-empty common part;

(C2) the digraph corresponding to the characteristic polynomial does not have additional cycles;

(C3) there exists a digraph mask corresponding to the characteristic polynomial;
(C4) there exists a digraph as a superposition operation on the digraph corresponding to the characteristic polynomial and a digraph mask in which all vertices have weights equal to 1 ;

(C5) in an extended digraph there are all paths from the source $s$ to the output $y$, which have the length $p=$ $2,3, \ldots, q+2$.

Proof. If conditions (C1) and (C2) are satisfied, then we can determine a digraph structure corresponding to a characteristic polynomial $\widetilde{d}(\lambda)$ in the form of (13). Otherwise, the realization does not exist. Conditions (C1) and $(\mathrm{C} 2)$ are presented in detail in Section 3.1. Then, if condition (C3) holds, then we can determine (by Proposition 2) $q+1$ possible digraph masks which guarantees the singularity of the solution. Condition (C3) is presented in detail in Section 3.2.

If conditions (C1)-(C3) are met, then by using a superposition operation on a digraph and the digraph mask we can determine all possible digraph structures. Then we choose digraphs in which all vertices have weights equal to 1 . Other solutions are rejected. Condition $(\mathrm{C} 4)$ is presented in detail in Section 3.3. Finally, to the digraph structures which met conditions $(\mathrm{C} 1)-(\mathrm{C} 4)$ we add the source vertex $s$ and the output vertex $y$. By connecting the source vertex $s$ with vertices $v_{1}, v_{2}, \ldots, v_{q+1}$ and vertices $v_{1}, v_{2}, \ldots, v_{q+1}$ with the output vertex $y$, we can determine all paths from a source to an output. This type of connection guarantees that all polynomial coefficients $b_{q}$ appear in (19). Condition (C5) is presented in detail in Section 3.4. If conditions (C1)-(C5) are satisfied, then 
there exists a digraph structure which corresponds to a transfer function in the form of (11).

It should be noted that in Section 3.4 only two types of connection between the source vertex $s$ and the output vertex $y$ are shown. This type of connection guarantees that we will always be able to determine a digraph structure that corresponds to the transfer function (11). In general, there are many ways to connect the source $s$ with the output $y$. Occasionally, there is a digraph structure corresponding to the transfer function (11), but we cannot calculate the weight values. In this case, we must reject this structure.

\section{Numerical examples}

Find a minimal realization of the transfer function

$$
T(s)=\frac{b_{3} s^{1.8}+b_{2} s^{1.2}+b_{1} s^{0.6}+b_{0}}{-a_{2} s^{1.2}-a_{1} s^{0.6}-a_{0}} .
$$

Solution: Assuming that $\lambda=s^{0.6}$, we can rewrite the transfer function (23) in the following form:

$$
T(\lambda)=\frac{b_{3} \lambda^{3}+b_{2} \lambda^{2}+b_{1} \lambda+b_{0}}{-a_{2} \lambda^{2}-a_{1} \lambda-a_{0}} .
$$

After multiplying the denominator of (24) by $\lambda^{-q+1}=$ $\lambda^{-4}$, we obtain the following characteristic polynomial:

$$
d(\lambda)=-a_{2} \lambda^{-2}-a_{1} \lambda^{-3}-a_{0} \lambda^{-4},
$$

and present it in a temporary form,

$$
\begin{aligned}
\tilde{d}(\lambda) & =1+d(\lambda) \\
& =1-a_{2} \lambda^{-2}-a_{1} \lambda^{-3}-a_{0} \lambda^{-4} \\
& =\underbrace{\left(1-a_{2} \lambda^{-2}\right)}_{B_{2}} \cup \underbrace{\left(1-a_{1} \lambda^{-3}\right)}_{B_{1}} \cup \underbrace{\left(1-a_{0} \lambda^{-4}\right)}_{B_{0}},
\end{aligned}
$$

which is needed to draw the digraph.

For each simple binomial $B_{0}, B_{1}$ and $B_{2}$, using Proposition 1, we create digraph representations (Figs.11(a) 11(c)].

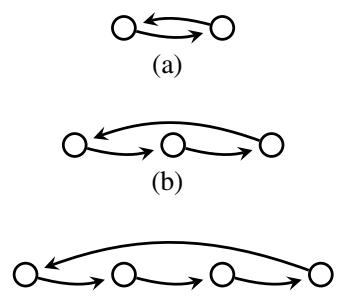

(c)

Fig. 11. Digraph corresponding to binomial: $B_{2}$ (a), $B_{1}$ (b), $B_{0}(\mathrm{c})$.

Now we can create all digraph realizations of the characteristic polynomial (26). One of the possible digraph structures is presented in Fig. 12

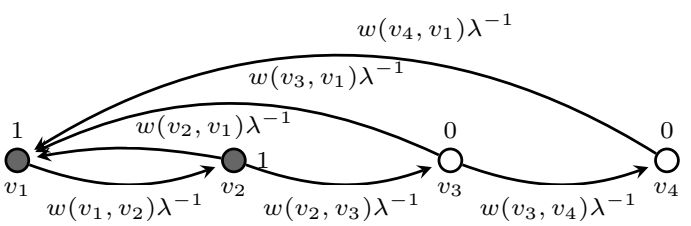

Fig. 12. One of the possible digraph structures corresponding to (26.

From the digraph we can write the following set of equations:

$$
\left\{\begin{array}{l|ll}
\lambda^{-2} & w\left(v_{1}, v_{2}\right) \cdot w\left(v_{2}, v_{1}\right) & =a_{2} \\
\lambda^{-3} & w\left(v_{1}, v_{2}\right) \cdot w\left(v_{2}, v_{3}\right) \cdot w\left(v_{3}, v_{1}\right) & =a_{1} \\
\lambda^{-4} & w\left(v_{1}, v_{2}\right) \cdot w\left(v_{2}, v_{3}\right) \cdot w\left(v_{3}, v_{4}\right) \cdot w\left(v_{4}, v_{1}\right)=a_{0} .
\end{array}\right.
$$

After solving them, we obtain the following arc weights:

$$
\begin{gathered}
w\left(v_{1}, v_{2}\right)=1, \quad w\left(v_{2}, v_{3}\right)=1, \\
w\left(v_{3}, v_{4}\right)=1, \quad w\left(v_{2}, v_{1}\right)=a_{2}, \\
w\left(v_{3}, v_{1}\right)=a_{1}, \quad w\left(v_{4}, v_{1}\right)=a_{0} .
\end{gathered}
$$

We can write the state matrix in the following form:

$$
\begin{aligned}
\mathbf{A} & =\left[\begin{array}{cccc}
0 & w\left(v_{2}, v_{1}\right) & w\left(v_{3}, v_{1}\right) & w\left(v_{4}, v_{1}\right) \\
w\left(v_{1}, v_{2}\right) & 0 & 0 & 0 \\
0 & w\left(v_{2}, v_{3}\right) & 0 & 0 \\
0 & 0 & w\left(v_{3}, v_{4}\right) & 0
\end{array}\right] \\
& =\left[\begin{array}{cccc}
0 & a_{2} & a_{1} & a_{0} \\
1 & 0 & 0 & 0 \\
0 & 1 & 0 & 0 \\
0 & 0 & 1 & 0
\end{array}\right]
\end{aligned}
$$

It should be noted that the set of equations has many possible solutions. The proposed solution 27 is only one of them.

In the next step, using Proposition 2, we must determine (see Fig. 13) a set of digraph masks consisting of four vertices. Three of them have weights equal to 1 and one of them has zero weight.

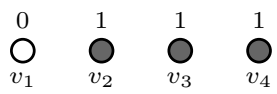

(a)

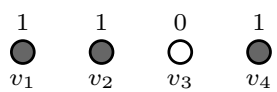

(c)

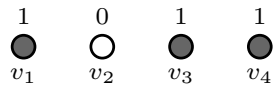

(b)
Fig. 13. All possible digraph masks. 
Then, using (15), we can determine all possible realizations of the characteristic polynomial (25) as a combination of the digraph representation presented, for example, in Fig. 12 and the digraph mask presented in Fig. 13. After this, we obtain four possible realizations
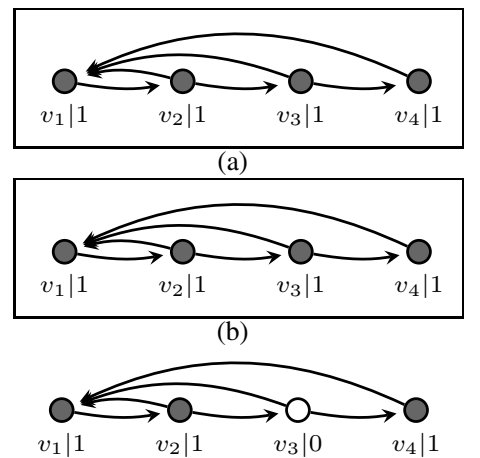

(c)

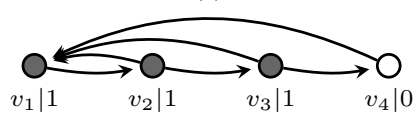

(d)

Fig. 14. All possible digraphs corresponding to 25].

(Fig. 14). It should be note that only two digraph structures presented in Figs. 14(a) and (b) satisfy the characteristic polynomial 25).

By using the digraph structure presented in Figs. 14(a) and (b), we can write the singular matrix in the following form:

$$
\mathbf{E}_{1}=\left[\begin{array}{cccc}
0 & 0 & 0 & 0 \\
0 & 1 & 0 & 0 \\
0 & 0 & 1 & 0 \\
0 & 0 & 0 & 1
\end{array}\right], \quad \mathbf{E}_{2}=\left[\begin{array}{cccc}
1 & 0 & 0 & 0 \\
0 & 0 & 0 & 0 \\
0 & 0 & 1 & 0 \\
0 & 0 & 0 & 1
\end{array}\right]
$$

In the second step, we should determine matrices $\mathbf{B}$ and C. For this purpose, we must multiply the numerator of the transfer function (24) by $\lambda^{-3}$. Then we obtain the following polynomial:

$$
n(\lambda)=b_{3}+b_{2} \lambda^{-1}+b_{1} \lambda^{-2}+b_{0} \lambda^{-3},
$$

and we expand the digraph created in the first step (Figs. 14(a) and 14(b)). We have two possible structures, which are considered in Sections 4.1 and 4.2.

4.1. Case 1. In this case, we connect the source vertex $s_{1}$ corresponding to matrix $\mathbf{B}$ with the vertex $v_{1}$ or $v_{2}$ belonging to a set of common parts of the digraph presented in Fig. 12, and the vertex $v_{1}, v_{2}, v_{3}, v_{4}$ with the output vertex $y_{1}$ corresponding to matrix $\mathbf{C}$. Then we determine all paths from the source vertex $s_{1}$ to the output vertex $y_{1}$. We obtain two possible digraph structures.

Digraph 1 -connect vertex $s_{1}$ with vertex $v_{1}$ : After this connection, we obtain a digraph presented in Fig. 15(a) Then, using the created digraph, we can write a set of equations in the form

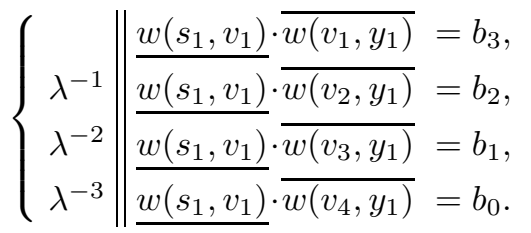

Solving them, we can write an input and output matrix in the following form:

$$
\begin{aligned}
& \mathbf{B}=\left[\begin{array}{c}
\frac{w\left(s_{1}, v_{1}\right)}{0} \\
0 \\
0
\end{array}\right] \\
& \mathbf{C}=\left[\frac{b_{3}}{\underline{w\left(s_{1}, v_{1}\right)}} \frac{b_{2}}{w\left(s_{1}, v_{1}\right)} \frac{b_{1}}{w\left(s_{1}, v_{1}\right)} \frac{b_{0}}{w\left(s_{1}, v_{1}\right)}\right] .
\end{aligned}
$$

The desired realization $\left(\mathbf{E}_{1}, \mathbf{A}, \mathbf{B}, \mathbf{C}\right)$ of 23 is given by (29), 28) and (31). It should be noted that realization is positive if $w\left(s_{1}, v_{1}\right) \in \mathbb{R}_{+}$.

Digraph 2-connect vertex $s_{1}$ with vertex $v_{2}$ : After this connection, we obtain the digraph presented in Fig. 15(b) Then, using the created digraph, we can write the following set of equations:

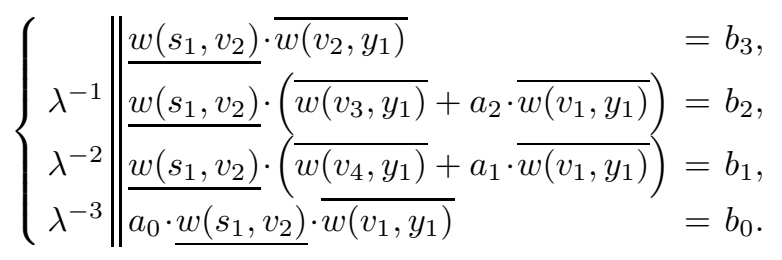

Solving them, we can write an input and output matrix in the following form:

$$
\begin{aligned}
& \widetilde{\mathbf{B}}=\left[\begin{array}{c}
0 \\
\frac{w\left(s_{1}, v_{2}\right)}{0} \\
0
\end{array}\right] \text {, } \\
& \widetilde{\mathbf{C}}=\left[\overline{w\left(v_{1}, y_{1}\right)} \overline{w\left(v_{2}, y_{1}\right)} \overline{w\left(v_{3}, y_{1}\right)} \overline{w\left(v_{4}, y_{1}\right)}\right],
\end{aligned}
$$

where

$$
\begin{aligned}
& \overline{w\left(v_{1}, y_{1}\right)}=\frac{b_{0}}{a_{0} \cdot \frac{w\left(s_{1}, v_{2}\right)}{b_{3}}}, \\
& \overline{w\left(v_{2}, y_{1}\right)}=\frac{\frac{w\left(s_{1}, v_{2}\right)}{a_{0} \cdot b_{2}-a_{2} \cdot b_{0}}}{a_{0} \cdot \underline{w\left(s_{1}, v_{2}\right)}},
\end{aligned}
$$




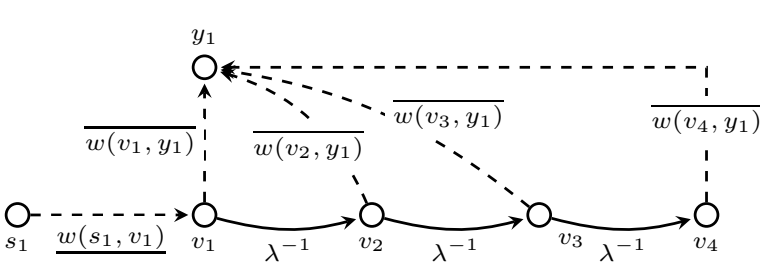

(a)

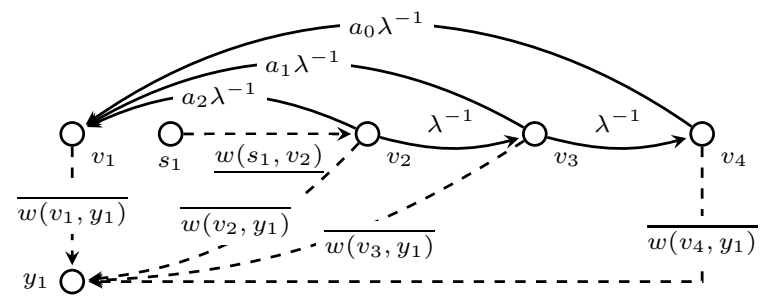

(b)

Fig. 15. Realization of (30) in Case 1: Digraph 1 (a), Digraph 2 (b).

$$
\overline{w\left(v_{4}, y_{1}\right)}=\frac{a_{0} \cdot b_{1}-a_{1} \cdot b_{0}}{a_{0} \cdot \underline{w\left(s_{1}, v_{2}\right)}} \text {. }
$$

The desired realization $\left(\mathbf{E}_{2}, \mathbf{A}, \widetilde{\mathbf{B}}, \widetilde{\mathbf{C}}\right)$ of 23 is given by (29), (28) and (32). It should be noted that the realization is positive if $w\left(s_{1}, v_{2}\right) \in \mathbb{R}_{+}$and the coefficients of the polynomials (25) and (30) satisfy the following conditions:

$$
\begin{aligned}
& a_{0} \cdot b_{2}-a_{2} \cdot b_{0}>0, \\
& a_{0} \cdot b_{1}-a_{1} \cdot b_{0}>0 .
\end{aligned}
$$

4.2. Case 2. In this case, we connect the source vertex $s_{1}$ corresponding to matrix $\mathbf{B}$ with the vertex $v_{1}, v_{2}, v_{3}$ and $v_{4}$, and the vertex $v_{1}$ or $v_{2}$ belonging to the set of common parts of the digraph presented in Fig. 12 with the output vertex $y_{1}$ corresponding to matrix $\mathbf{C}$. Then, we determine all paths from the source vertex $s_{1}$ to the output vertex $y_{1}$. We obtain two possible digraph structures.

Digraph 1 -connect vertex $v_{1}$ with vertex $y_{1}$ : After this connection, we obtain the digraph presented in Fig. 16(a) Then, using the created digraph, we can write the following set of equations:

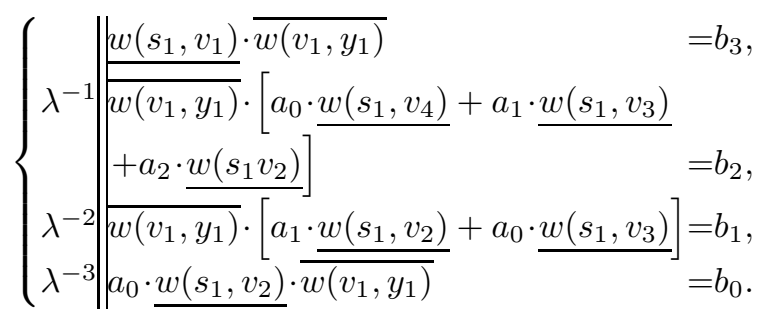

Solving them, we can write an input and output matrix in the following form:

$$
\overline{\mathbf{B}}=\left[\frac{\frac{w\left(s_{1}, v_{1}\right)}{w\left(s_{1}, v_{2}\right)}}{\frac{w\left(s_{1}, v_{3}\right)}{w\left(s_{1}, v_{4}\right)}}\right],
$$

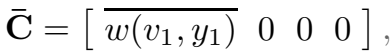

where

$$
\begin{aligned}
& \underline{w\left(s_{1}, v_{1}\right)}=\frac{b_{3}}{\overline{w\left(v_{1}, y_{1}\right)}}, \\
& \underline{w\left(s_{1}, v_{2}\right)}=\frac{b_{0}}{a_{0} \cdot \overline{w\left(v_{1}, y_{1}\right)}}, \\
& \underline{w\left(s_{1}, v_{3}\right)}=\frac{a_{0} \cdot b_{1}-a_{1} \cdot b_{0}}{a_{0}^{2} \cdot \overline{w\left(v_{1}, y_{1}\right)}}, \\
& \frac{w\left(s_{1}, v_{4}\right)}{=}=\frac{a_{0} \cdot\left(a_{0} \cdot b_{2}-a_{2} \cdot b_{0}\right)-a_{1} \cdot\left(a_{0} \cdot b_{1}-a_{1} \cdot b_{0}\right)}{a_{0}^{3} \cdot \overline{w\left(v_{1}, y_{1}\right)}} \text {. }
\end{aligned}
$$

The desired realization $\left(\mathbf{E}_{1}, \mathbf{A}, \overline{\mathbf{B}}, \overline{\mathbf{C}}\right)$ of $(23)$ is given by (29), (28) and (33). It should be noted that the realization is positive if $\overline{w\left(v_{1}, y_{1}\right)} \in \mathbb{R}_{+}$and the coefficients of the polynomial (25) and (30) satisfy the following conditions:

$$
\begin{gathered}
a_{0} \cdot b_{1}-a_{1} \cdot b_{0}>0 \\
a_{0} \cdot\left(a_{0} \cdot b_{2}-a_{2} \cdot b_{0}\right)-a_{1} \cdot\left(a_{0} \cdot b_{1}-a_{1} \cdot b_{0}\right)>0 .
\end{gathered}
$$

Digraph 2-connect vertex $v_{2}$ with vertex $y_{1}$ : After this connection, we obtain the digraph presented in Fig. 16(b), Then, using the created digraph, we can write a set of equations:

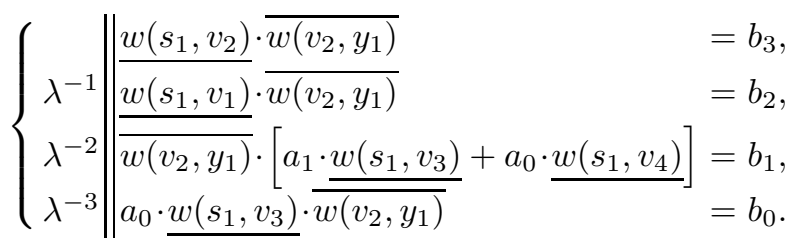

Solving them, we can write an input and output matrix in the following form:

$$
\begin{aligned}
& \check{\mathbf{B}}=\left[\frac{\frac{w\left(s_{1}, v_{1}\right)}{w\left(s_{1}, v_{2}\right)}}{\frac{w\left(s_{1}, v_{3}\right)}{w\left(s_{1}, v_{4}\right)}}\right], \\
& \check{\mathbf{C}}=\left[\begin{array}{lll}
0 \overline{w\left(v_{2}, y_{1}\right)} & 0 & 0
\end{array}\right],
\end{aligned}
$$




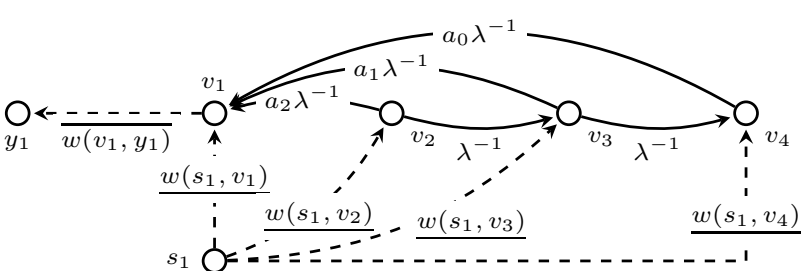

(a)

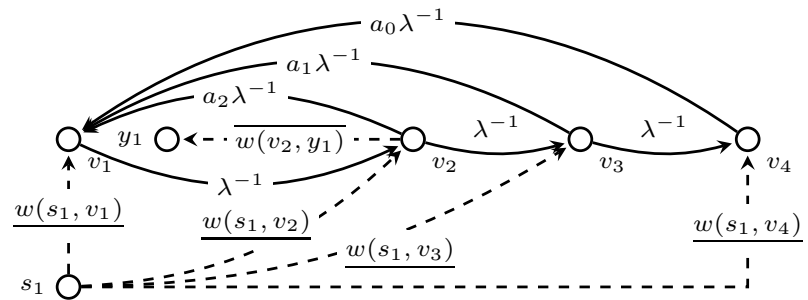

(b)

Fig. 16. Realization of (30) in Case 2: Digraph 1 (a), Digraph 2 (b).

where

$$
\begin{aligned}
& \frac{w\left(s_{1}, v_{1}\right)}{=}=\frac{b_{2}}{\overline{w\left(v_{2}, y_{1}\right)}}, \\
& \underline{w\left(s_{1}, v_{2}\right)}=\frac{b_{3}}{\overline{w\left(v_{2}, y_{1}\right)}}, \\
& \underline{w\left(s_{1}, v_{3}\right)}=\frac{b_{0}}{a_{0} \cdot \overline{w\left(v_{2}, y_{1}\right)}}, \\
& \underline{w\left(s_{1}, v_{4}\right)}=\frac{a_{0} \cdot b_{1}-a_{1} \cdot b_{0}}{a_{0}^{2} \cdot \overline{w\left(v_{2}, y_{1}\right)}}
\end{aligned}
$$

The desired realization $\left(\mathbf{E}_{2}, \mathbf{A}, \check{\mathbf{B}}, \check{\mathbf{C}}\right)$ of $(23)$ is given by (29), (28) and (34). It should be noted that the realization is positive if $\overline{w\left(v_{2}, y_{1}\right)} \in \mathbb{R}_{+}$and the coefficients of the polynomials (25) and (30) satisfy the following conditions:

$$
a_{0} \cdot b_{1}-a_{1} \cdot b_{0}>0
$$

\section{Concluding remarks}

A method for computation of a minimal realization of a given proper transfer function of fractional positive descriptor one-dimensional continuous-time linear systems has been proposed. Sufficient conditions for the existence of a minimal set of realizations of a given proper transfer function have been established. A method based on one-dimensional digraph theory for the computation of minimal realizations has been proposed. It should be noted that in many research studies we can find a canonical form of the system, i.e., a constant matrix form, which satisfies the system described by the transfer function. With the use of this form, we are able to write only one realization of the system, while there exists a set of solutions. In this paper a solution to the problem of finding a set of possible minimal positive realizations has been proposed. Additionally, the possibility of an easy implementation in the form of a computer algorithm using $\mathrm{CPU}$ and/or GPU processors is the strength of this digraph method. The effectiveness of the algorithm has been illustrated with some numerical examples. An extension of this study to fractional descriptor discrete-time linear systems and to a fractional descriptor hybrid model is possible.

Further work includes extension of the proposed method to find all possible solutions for other structures of matrix $\mathbf{E}$ and extension of the algorithm to find the class of electrical circuits corresponding to the transmission matrix.

\section{References}

Bang-Jensen, J. and Gutin, G. (2009). Digraphs: Theory, Algorithms and Applications, Springer-Verlag, London.

Benvenuti, L. and Farina, L. (2004). A tutorial on the positive realization problem, IEEE Transactions on Automatic Control 49(5): 651-664.

Berman, A. and Plemmons, R.J. (1979). Nonnegative Matrices in the Mathematical Sciences, SIAM, London.

Caputo, M. (1967). Linear models of dissipation whose $q$ is almost frequency independent-II, Geophysical Journal International 13(5): 529, DOI: 10.1111/ j.1365-246X.1967.tb02303.x.

Dai, L. (Ed.) (1989). System Analysis via Transfer Matrix, Springer, Berlin/Heidelberg, DOI: 10.1007/BFb0002482.

Das, S. (2011). Functional Fractional Calculus, Springer, Berlin/Heidelberg, DOI: 10.1007/978-3-642-20545-3.

Dodig, M. and Stoi, M. (2009). Singular systems, state feedback problem, Linear Algebra and Its Applications 431(8): 1267-1292, DOI:10.1016/j.laa.2009.04.024.

Farina, L. and Rinaldi, S. (2000). Positive Linear Systems: Theory and Applications, Wiley-Interscience, New York, NY.

Fornasini, E. and Valcher, M.E. (1997). Directed graphs, 2D state models, and characteristic polynomials of irreducible matrix pairs, Linear Algebra and Its Applications 263: 275-310.

Fornasini, E. and Valcher, M.E. (2005). Controllability and reachability of 2D positive systems: A graph theoretic approach, IEEE Transactions on Circuits and Systems I 52(3): 576-585.

Godsil, C. and Royle, G. (2001). Algebraic Graph Theory, Springer Verlag, New York, NY.

Guang-Ren, D. (2010). Analysis and Design of Descriptor Linear Systems, Springer, New York, NY, DOI: 10.1007/978-1-4419-6397-0. 
Horn, R.A. and Johnson, C.R. (1991). Topics in Matrix Analysis, Cambridge University Press, Cambridge.

Hryniów, K. and Markowski, K.A. (2014). Parallel digraphs-building algorithm for polynomial realisations, Proceedings of 15th International Carpathian Control Conference (ICCC), Velke Karlovice, Czech Republic, pp. 174-179, DOI: 10.1109/CarpathianCC.2014.6843592.

Hryniów, K. and Markowski, K.A. (2015). Optimisation of digraphs creation for parallel algorithm for finding a complete set of solutions of characteristic polynomial, Proceedings of the 20th International Conference on Methods and Models in Automation and Robotics, MMAR 2015, Miedzyzdroje, Poland, pp. 1139-1144, DOI: 10.1109/MMAR.2015.7284039.

Hryniów, K. and Markowski, K.A. (2016a). Classes of digraph structures corresponding to characteristic polynomials, in R. Szewczyk et al. (Eds.), Challenges in Automation, Robotics and Measurement Techniques: Proceedings of Automation 2016, Warsaw, Poland, Springer International Publishing, Cham, pp. 329-339, DOI: 10.1007/978-3-319-29357-8_30.

Hryniów, K. and Markowski, K.A. (2016b). Parallel digraphs-building computer algorithm for finding a set of characteristic polynomial realisations of dynamic system, Journal of Automation, Mobile Robotics and Intelligent Systems 10(03): 38-51, DOI: 10.14313/JAMRIS_3-2016/23.

Ionescu, C.M., Kosinski, W. and De Keyser, R. (2010). Viscoelasticity and fractal structure in a model of human lungs, Archives of Mechanics 62(1): 21-48.

Kaczorek, T. (2001). Positive $1 D$ and 2D Systems, Springer Verlag, London.

Kaczorek, T. (2007). Polynomial and Rational Matrices, Springer Verlag, London.

Kaczorek, T. (2011). Singular fractional linear systems and electrical circuits, International Journal of Applied Mathematics and Computer Science 21(2): 379-384, DOI: 10.2478/v10006-011-0028-8.

Kaczorek, T. and Sajewski, L. (2014). The Realization Problem for Positive and Fractional Systems, Springer International Publishing, Berlin, DOI: 10.1007/978-3-319-04834-5.

Kublanovskaya, V.N. (1983). Analysis of singular matrix pencils, Journal of Soviet Mathematics 23(1): 1939-1950, DOI: 10.1007/BF01093276.

Lewis, F. (1984). Descriptor systems: Decomposition into forward and backward subsystems, IEEE Transactions on Automatic Control 29(2): 167-170, DOI: 10.1109/TAC.1984.1103467.

Lewis, F.L. (1986). A survey of linear singular systems, Circuits, Systems and Signal Processing 5(1): 3-36, DOI: 10.1007/BF01600184.

Luenberger, D.G. (1979). Introduction to Dynamic Systems: Theory, Models, and Applications, Wiley, New York, NY.

Machado, J. and Lopes, A.M. (2015). Fractional state space analysis of temperature time series, Fractional Calculus and Applied Analysis 18(6): 1518-1536.
Machado, J., Mata, M.E. and Lopes, A.M. (2015). Fractional state space analysis of economic systems, Entropy 17(8): 5402-5421.

Magin, R., Ortigueira, M.D., Podlubny, I. and Trujillo, J. (2011). On the fractional signals and systems, Signal Processing 91(3): 350-371.

Markowski, K.A. (2016). Digraphs structures corresponding to minimal realisation of fractional continuous-time linear systems with all-pole and all-zero transfer function, 2016 IEEE International Conference on Automation, Quality and Testing, Robotics (AQTR), Cluj-Napoca, Romania, pp. 1-6, DOI: 10.1109/AQTR.2016.7501367.

Markowski, K.A. (2017a). Determination of minimal realisation of one-dimensional continuous-time fractional linear system, International Journal of Dynamics and Control 5(1): 40-50, DOI: 10.1007/s40435-016-0232-3.

Markowski, K.A. (2017b). Realisation of continuous-time (fractional) descriptor linear systems, in $\mathrm{R}$. Szewczyk et al. (Eds.), Automation 2017, Springer International Publishing, Cham, pp. 204-214, DOI: 10.1007/978-3-319-54042-9_19.

Markowski, K.A. (2017c). Realisation of linear continuous-time fractional singular systems using digraph-based method: First approach, Journal of Physics: Conference Series 783(1): 012052, DOI: 10.1088/1742-6596/783/1/012052.

Markowski, K.A. (2018). Classes of digraphs structures with weights corresponding to 1D fractional systems, International Conference on Automation, Quality and Testing, Robotics, AQTR 2018, Cluj-Napoca, Romania, (submitted).

Markowski, K.A. and Hryniów, K. (2017a). Expansion of digraph size of 1-D fractional system with delay, in A. Babiarz et al. (Eds.), Theory and Applications of Non-integer Order Systems, Springer International Publishing, Cham, pp. 467-476, DOI: 10.1007/978-3-319-45474-0_41.

Markowski, K.A. and Hryniów, K. (2017b). Finding a set of (A, B, C, D) realisations for fractional one-dimensional systems with digraph-based algorithm, in A. Babiarz et al. (Eds.), Theory and Applications of Non-integer Order Systems, Springer International Publishing, Cham, pp. 357-368, DOI: 10.1007/978-3-319-45474-0_32.

Miller, K. and Ross, B. (1993). An Introduction to the Fractional Calculus and Fractional Differential Equations, Wiley, New York, NY.

Mitkowski, W. (2008). Dynamical properties of Metzler systems, Bulletin of the Polish Academy of Sciences: Technical Sciences 56(4): 309-312.

Muresan, C.I., Dulf, E.H. and Prodan, O. (2016a). A fractional order controller for seismic mitigation of structures equipped with viscoelastic mass dampers, Journal of Vibration and Control 22(8): 1980-1992, DOI: $10.1177 / 1077546314557553$.

Muresan, C.I., Dutta, A., Dulf, E.H., Pinar, Z., Maxim, A. and Ionescu, C.M. (2016b). Tuning algorithms for fractional order internal model controllers for time delay processes, 
International Journal of Control 89(3): 579-593, DOI: 10.1080/00207179.2015.1086027.

Nishimoto, K. (1984). Fractional Calculus, Decartess Press, Koriama.

Ortigueira, M.D. (2011). Fractional Calculus for Scientists and Engineers, Academic Press, Springer, Dordrecht, DOI: 10.1007/978-94-007-0747-4.

Petras, I., Sierociuk, D. and Podlubny, I. (2012). Identification of parameters of a half-order system, IEEE Transactions on Signal Processing 60(10): 5561-5566.

Podlubny, I. (1999). Fractional Differential Equations, Academic Press, San Diego, CA.

Podlubny, I., Skovranek, T. and Datsko, B. (2014). Recent advances in numerical methods for partial fractional differential equations, 2014 15th International Carpathian Control Conference (ICCC), Velke Karlovice, Czech Republic, pp. 454-457.

Sajewski, L. (2012). Positive realization of fractional continuous-time linear systems with delays, Pomiary Automatyka Robotyka 2: 413-417.

Sikora, B. (2016). Controllability criteria for time-delay fractional systems with a retarded state, International Journal of Applied Mathematics and Computer Science 26(3): 521-531, DOI: 10.1515/amcs-2016-0036.
Vandoorn, T.L., Ionescu, C.M., De Kooning, J.D.M., De Keyser, R. and Vandevelde, L. (2013). Theoretical analysis and experimental validation of single-phase direct versus cascade voltage control in islanded microgrids, IEEE Transactions on Industrial Electronics 60(2): 789-798.

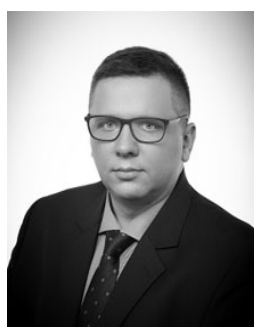

Konrad A. Markowski (born 1977, Warsaw, Poland) received his MSc and $\mathrm{PhD}$ degrees from the Faculty of Electrical Engineering at the Warsaw University of Technology in 2003 and 2008, respectively. Since 2008 he has been an assistant professor. In 2009 he became an IEEE member and in 2015 an IEEE senior member. His research interest cover systems theory and automatic control, especially multidimensional systems, positive multidimensional systems, fractional systems, and descriptor systems. Recently, he has been particularly interested in using graph theory and multiprocessing in solving numerical problems in the field of control theory. Also, he has become interested in the development of control technology for rockets systems, aerospace systems and space systems. As a result, in 2017 he started work as a senior project manager in the field of defence and aerospace technology. Participation in business projects has allowed him to establish some new directions of his scientific work.

Received: 5 February 2017

Revised: 12 June 2017

Accepted: 9 August 2017 